\title{
Cognitive Impairment and Recovery in Meningiomas and Low-Grade Gliomas
}

\author{
Jiri Lahti1,2*, Tiia Saunamäki ${ }^{3}$, Jarmo Salo ${ }^{4,5}$, Asko Niemelä ${ }^{6,7}$, Mervi Jehkonen ${ }^{1}$ \\ ${ }^{1}$ The Unit of Psychology, Faculty of Social Sciences, University of Tampere, Tampere, Finland \\ ${ }^{2}$ Department of Medical Rehabilitation, Oulu University Hospital, Oulu, Finland \\ ${ }^{3}$ Department of Neurology and Rehabilitation, Tampere University Hospital, Tampere, Finland \\ ${ }^{4}$ Department of Children and Adolescents, Oulu University Hospital, Oulu, Finland \\ ${ }^{5}$ PEDEGO Research Unit and Medical Research Center Oulu, University of Oulu, Oulu, Finland \\ ${ }^{6}$ Department of Psychiatry, Oulu University Hospital, Oulu, Finland \\ ${ }^{7}$ Department of Neurosurgery, Oulu University Hospital, Oulu, Finland \\ Email: *jiri.lahti@gmail.com
}

How to cite this paper: Lahti, J., Saunamäki, T., Salo, J., Niemelä, A. and Jehkonen, M. (2018) Cognitive Impairment and Recovery in Meningiomas and Low-Grade Gliomas. Journal of Behavioral and Brain Science, 8, 473-484. https://doi.org/10.4236/jbbs.2018.88029

Received: June 26, 2018

Accepted: August 7, 2018

Published: August 10, 2018

Copyright ( 92018 by authors and Scientific Research Publishing Inc. This work is licensed under the Creative Commons Attribution International License (CC BY 4.0).

http://creativecommons.org/licenses/by/4.0/

\section{(c) (i) Open Access}

\begin{abstract}
This study aims to elucidate the nature of cognitive deficits caused by intracranial tumors, as well as to examine how a surgical operation of the tumor may affect tumor-induced cognitive deficits. The patient group included 43 individuals with meningioma or low-grade glioma admitted to a surgical operation of the tumor. Neuropsychological examination was conducted preoperatively, as well as three and 12 months postoperatively. The control group comprised 31 healthy subjects. In the tumor patients, preoperative cognitive performance was compromised in several cognitive domains as compared to the controls. The tumor patients with frontal and large tumors showed impairment virtually across all cognitive domains. Postoperatively, the cognitive performance of the meningioma and the small tumor group improved in all domains, with the performance of the low-grade glioma group and the large tumor group reflecting more modest cognitive improvement. Most of this improvement did not emerge until the 12 months follow-up. Cognitive impairment due to an intracranial tumor is diffuse affecting most cognitive domains. Cognitive recovery after the surgery is more noticeable in patients with meningiomas and small tumors, and the recovery will require a minimum of one year time-wise. This evidence is of significant value when planning both clinical treatment and rehabilitation of intracranial tumor patients.
\end{abstract}

\section{Keywords}

Intracranial Tumor, Cognition, Neuropsychological Disorder, Neuropsychology, Neurosurgery 


\section{Introduction}

Intracranial tumors commonly result in both neurological and neuropsychological deficits. Namely, infiltration and pressure from tumoral tissue disturb the normal functionality of the brain, which in turn is associated with a deterioration of several cognitive functions, such as those pertaining to memory, visuospatial, verbal and more broadly, executive operations [1] [2].

Tumors located in the dominant hemisphere of the brain, usually the left hemisphere (LH), are commonly linked to aphasic disorders as well as other forms of verbal deficits [3] [4]. By contrast, tumors of the right hemisphere (RH) are frequently associated with visuospatial deficits [5] [6]. However, LH tumors are associated with milder impairment in verbal functions as contrasted with other LH-located lesions such as strokes [7]. Dominant hemisphere tumors have been found to induce more diffuse and severe cognitive disturbances as compared to non-dominant hemisphere tumors [3] [8] [9]. There is growing evidence indicating that instead of focal deficits based on the tumor location, intracranial tumors are more frequently linked to generalized and diffuse cognitive deficits, such as problems in executive and memory functions [10] [11] [12]. In addition, verbal impairments have been linked to both $\mathrm{LH}$ and $\mathrm{RH}$ tumors [8]. This has been attributed to the characteristics of intracranial tumors: their impact on the functionality of the brain is greater than that expected on the basis of tumor location alone [2] [13].

The histological type of the tumor is linked to the severity of the cognitive impairment, with higher-grade tumors being associated with more severe cognitive impairment than lower-grade tumors [3] [14]. In addition, the size of the tumor is associated with cognitive impairment [14]: rapidly progressing tumors are linked to more severe cognitive symptoms than slowly progressing ones due to the mass effect of the tumoral tissue [2] [15]. Possible explanations for this may include the reorganization of functional networks found in slowly growing tumors, which is capable of compensating for the effects of the tumor on cognitive skills [16].

In order to obtain a reliable estimate of how the intracranial tumor itself, as well as its treatment methods, influence cognitive functions, neuropsychological assessment must be conducted both pre- and post-operatively [2] [17]. The surgical operation of the tumor may be associated with either improvement [11] [18] or decline of cognitive functions [5]. Additionally, the deterioration of executive functions with an improvement of memory functions has been reported [14]. It is thus possible that the outcome of the surgery depends on the histology of the tumor, since meningioma patients are found to improve their cognitive functioning after surgery [19].

Currently, there is only a handful of post-treatment studies in which the effects of the tumor treatment on cognitive functions has been studied systematically, in which a control group has been included, as well as a more extensive neuropsychological assessment battery in addition to mere screening tests, such 
as the Mini-Mental State Examination (MMSE) [20]. To address this gap in the literature, the aim of the present study was four-fold, to carefully delineate the cognitive performance of the meningioma and low-grade glioma patients preand post-operatively. Specifically, we set out to examine: 1) whether intracranial tumors are linked to specific or general neuropsychological deficits, 2) how tumor malignancy, size, and location of the tumor are related to cognitive functions, 3) how surgical treatment affects cognitive functions, and 4) how patients recover three and 12 months after the surgery.

\section{Materials and Methods}

\subsection{Subjects}

The study included all patients with meningioma or glioma treated with partial or complete resection of the tumor at the Clinic of Neurosurgery in Oulu University Hospital during two years. Patients were excluded from the study if their chronological age was either under 16 or over 75 years, they had tumor metastases or their physical condition was too weak hampering the participation in neuropsychological examination. After these criteria were met, the neuropsychological examination was conducted for 43 patients. Patients were assessed with a neuropsychological examination preoperatively, as well as three and 12 months postoperatively. Two patients dropped out before the three-month follow-up due to death. The enrolled patients did not receive any neuropsychological rehabilitation. The control group comprised 31 volunteers with no diagnosed neurological diseases age-matched to the clinical patients. The control group was administered an identical neuropsychological assessment to the tumor patients with the exception of the follow-up procedure, which was omitted. In addition, to screen for global cognitive impairment, those volunteers who were over 55 years and/or retired from work were submitted to a cognitive screening test. For this purpose, the MMSE [21] was used with the exclusion criteria of score of $<25$. Both the patient group and the control group were native finnish speakers. Demographic characteristics of the patient and control groups are presented in Table 1.

\subsection{Analysis of Neurological and Tumor Parameters}

Neurological examination was carried out by a physician. The histological grade of tumors was defined according to the World Health Organization (WHO) classification of tumors [22]. Either preoperative computerized tomography (CT) or magnetic resonance imaging (MRI) was conducted for all patients. CT or MRI images for sufficiently accurate analysis of tumor volume and location were not available for three patients. Patients were divided into subgroups on the basis of their tumor characteristics. Based on brain imaging, tumors were classified according to location (anterior/posterior) and hemisphere (LH/RH). Tumors that could not be classified according to these categories were classed either as undefined or bilateral. The volume of each tumor was calculated manually from 
Table 1. Demographic data and characteristics of the patient group and the control group.

\begin{tabular}{ccc}
\hline & Patients $(\mathrm{n}=43)$ & Controls $(\mathrm{n}=31)$ \\
\hline Age $(\mathrm{mean}, \mathrm{SD})^{*}$ & $46.3 \pm 12.5$ & $45.6 \pm 19.0$ \\
Sex $(\mathrm{M} / \mathrm{F})^{*}$ & $14 / 29$ & $10 / 21$ \\
Location of tumor: & & - \\
L/R/B & $21 / 14 / 5^{* *}$ & - \\
A/P/UD & $24 / 13 / 6$ & \\
Tumor diagnosis: & & - \\
Meningioma & 25 & - \\
Grade I-II glioma & 18 & \\
Tumor volume (median, IQR in ml) & $31.7(10.8-58.8)$ & \\
Treated with radiation therapy & & - \\
Meningioma & 2 & \\
Grade I-II glioma & 15 & \\
Treated with chemotherapy & & \\
Meningioma & & \\
Grade I-II glioma & 3 &
\end{tabular}

Abbreviations: $\mathrm{SD}=$ standard deviation; $\mathrm{M}=$ male; $\mathrm{F}=$ female; $\mathrm{L}=$ left; $\mathrm{R}=$ right; $\mathrm{B}=$ bilateral; $\mathrm{A}=$ anterior; $\mathrm{P}=$ posterior; $\mathrm{UD}=$ undefined; $\mathrm{IQR}=$ interquartile range. ${ }^{\star}$ The patient and the control group did not differ significantly in age (Mann-Whitney $U$ Test; $U=619.5, p=0.61$ ) or gender (Chi-square test; $\chi^{2}=0.01$, $\mathrm{p}=0.98) .{ }^{*} \mathrm{CT}$ or MRI images for sufficiently accurate analysis of tumor volume and lateral location were not available for three patients.

the CT or MRI images, and each tumor was then classified according to size (small/large) on the basis of the median (small $<31,70 \mathrm{ml} \leq$ large) of the tumor volume. CT was used predominantly and the neuroradiologist was consulted.

\subsection{Neuropsychological Examination}

Verbal functions were assessed using the Similarities subtest of the Wechsler Adult Intelligence Scale (WAIS) [23], while visuospatial functions were examined using the Block Design subtest of the WAIS. The Babcock Story [24] together with the Word List Recall Task [25] was administered in order to obtain an estimate of the subjects' immediate and delayed memory performance. Executive and attentional functions were assessed both by measuring the total time of reading the color-word interference trial from the Golden version of the Stroop test [26] as well as the total time of the Serial Seven Subtraction test [27].

\subsection{Statistical Analysis}

Statistical analyses were conducted using non-parametric tests due to the variables failing to meet the assumptions of normality. Medians and quartiles were used as descriptive values. If the subject failed to complete the Stroop test or the Serial Subtraction test, the missing values were replaced with the maximum value from the sample, in order to include an estimate for cases representing weak 
performance into the analyses. The comparisons between the tumor groups and the controls were analyzed utilizing the two-tailed Kruskal-Wallis test, while the comparisons within the tumor groups at the follow-up were analyzed with the Wilcoxon Signed-Rank test. The statistical significance was set at $\mathrm{p}<0.05$ for both tests.

\section{Results}

The patient group underwent the neuropsychological assessment preoperatively, as well as three and 12 months postoperatively, while the control group underwent the assessment once. Results of the preoperative neuropsychological assessment are displayed in Table 2 and Table 3.

\subsection{Preoperative Assessment of Cognitive Functions: Effect of the Tumor Location, Size and Histological Type}

In general terms, the clinical patients showed significantly weaker performance in nearly all cognitive domains as compared to the controls. Comparisons between the tumor groups and the controls are presented in Table 4.

Relative to the controls, the anterior tumor group performed at a significantly lower level in all cognitive domains, whereas the posterior tumor group showed significant deficits in visuospatial, attentional, and delayed memory functions. Both the LH and RH tumor groups demonstrated significantly lower levels of

Table 2. Means, standard deviations and ranges of the raw scores at the preoperative neuropsychological assessment.

\begin{tabular}{|c|c|c|c|c|c|c|c|c|c|}
\hline Group & & Simil & Block & Word tot & Word del & Story imm & Story del & Serial & Stroop \\
\hline MNG & $(\mathrm{n}=25)$ & $\begin{array}{c}17.4 \pm 5.3 \\
(5-25)\end{array}$ & $\begin{array}{c}24.0 \pm 5.3 \\
(8-47)\end{array}$ & $\begin{array}{c}38.5 \pm 8.8 \\
(9-48)\end{array}$ & $\begin{array}{c}6.21 \pm 2.6 \\
(0-10)\end{array}$ & $\begin{array}{c}8.0 \pm 3.4 \\
(3-16)\end{array}$ & $\begin{array}{c}10.3 \pm 4.0 \\
(2-17)\end{array}$ & $\begin{array}{c}160.9 \pm 123.0 \\
(25-400)\end{array}$ & $\begin{array}{c}249.6 \pm 171.1 \\
(76-530)\end{array}$ \\
\hline LGG & $(\mathrm{n}=18)$ & $\begin{array}{c}16.7 \pm 3.6 \\
(10-23)\end{array}$ & $\begin{array}{l}29.8 \pm 12.4 \\
(6-47)\end{array}$ & $\begin{array}{c}40.0 \pm 6.1 \\
(28-49)\end{array}$ & $\begin{array}{c}6.4 \pm 2.3 \\
(0-10)\end{array}$ & $\begin{array}{c}7.2 \pm 3.9 \\
(2-16)\end{array}$ & $\begin{array}{c}9.3 \pm 4.0 \\
(1-16)\end{array}$ & $\begin{array}{c}140.1 \pm 119.3 \\
(30-400)\end{array}$ & $\begin{array}{c}210 \pm 156.0 \\
(82-530)\end{array}$ \\
\hline Small & $(\mathrm{n}=20)$ & $\begin{array}{c}17.5 \pm 5.0 \\
(5-23)\end{array}$ & $\begin{array}{c}26.4 \pm 9.7 \\
(10-47)\end{array}$ & $\begin{array}{c}40.0 \pm 9.4 \\
(9-49)\end{array}$ & $\begin{array}{c}6.2 \pm 2.8 \\
(0-10)\end{array}$ & $\begin{array}{c}8.1 \pm 4.2 \\
(2-16)\end{array}$ & $\begin{array}{c}11.1 \pm 4.3 \\
(2-17)\end{array}$ & $\begin{array}{c}150.4 \pm 125.9 \\
(25-400)\end{array}$ & $\begin{array}{c}228.6 \pm 168.9 \\
(76-530)\end{array}$ \\
\hline Large & $(\mathrm{n}=20)$ & $\begin{array}{c}16.7 \pm 4.5 \\
(8-25)\end{array}$ & $\begin{array}{l}26.0 \pm 13.1 \\
(6-47)\end{array}$ & $\begin{array}{c}37.7 \pm 6.0 \\
(27-47)\end{array}$ & $\begin{array}{c}6.4 \pm 2.3 \\
(0-10)\end{array}$ & $\begin{array}{c}7.1 \pm 3.0 \\
(2-11)\end{array}$ & $\begin{array}{c}8.6 \pm 3.7 \\
(1-16)\end{array}$ & $\begin{array}{c}162.3 \pm 123.4 \\
(25-400)\end{array}$ & $\begin{array}{c}232.4 \pm 159.5 \\
(98-530)\end{array}$ \\
\hline LH & $(\mathrm{n}=21)$ & $\begin{array}{c}16.8 \pm 5.3 \\
(5-23)\end{array}$ & $\begin{array}{l}28.2 \pm 12.5 \\
\quad(8-47)\end{array}$ & $\begin{array}{c}37.7 \pm 9.5 \\
(9-49)\end{array}$ & $\begin{array}{c}6.4 \pm 2.7 \\
(0-10)\end{array}$ & $\begin{array}{c}7.3 \pm 3.9 \\
(2-16)\end{array}$ & $\begin{array}{c}10.0 \pm 4.1 \\
(3-17)\end{array}$ & $\begin{array}{c}164.7 \pm 135.6 \\
(25-400)\end{array}$ & $\begin{array}{c}213.1 \pm 162.2 \\
(76-530)\end{array}$ \\
\hline RH & $(\mathrm{n}=14)$ & $\begin{array}{c}17.4 \pm 4.2 \\
(11-25)\end{array}$ & $\begin{array}{l}23.4 \pm 10.8 \\
\quad(6-47)\end{array}$ & $\begin{array}{c}40.0 \pm 5.6 \\
(28-48)\end{array}$ & $\begin{array}{c}6.4 \pm 2.6 \\
(0-10)\end{array}$ & $\begin{array}{c}7.9 \pm 4.0 \\
(2-16)\end{array}$ & $\begin{array}{c}9.7 \pm 4.4 \\
(1-16)\end{array}$ & $\begin{array}{c}122.4 \pm 101.0 \\
(25-400)\end{array}$ & $\begin{array}{c}225.5 \pm 147.4 \\
(82-530)\end{array}$ \\
\hline Anterior & $(\mathrm{n}=24)$ & $\begin{array}{c}16.0 \pm 5.2 \\
(5-23)\end{array}$ & $\begin{array}{c}23.3 \pm 9.7 \\
(6-47)\end{array}$ & $\begin{array}{c}38.6 \pm 8.7 \\
(9-48)\end{array}$ & $\begin{array}{c}6.3 \pm 2.5 \\
(0-9)\end{array}$ & $\begin{array}{c}7.4 \pm 4.0 \\
(2-16)\end{array}$ & $\begin{array}{c}9.4 \pm 4.4 \\
(1-17)\end{array}$ & $\begin{array}{c}162.8 \pm 124.5 \\
(25-400)\end{array}$ & $\begin{array}{c}242.4 \pm 163.1 \\
(76-530)\end{array}$ \\
\hline Posterior & $(\mathrm{n}=13)$ & $\begin{array}{c}18.7 \pm 3.3 \\
(12-24)\end{array}$ & $\begin{array}{l}29.8 \pm 12.1 \\
\quad(8-47)\end{array}$ & $\begin{array}{c}38.9 \pm 7.1 \\
(29-48)\end{array}$ & $\begin{array}{c}6.2 \pm 2.7 \\
(0-10)\end{array}$ & $\begin{array}{c}7.8 \pm 3.1 \\
(2-11)\end{array}$ & $\begin{array}{c}9.9 \pm 3.5 \\
(4-16)\end{array}$ & $\begin{array}{c}124.6 \pm 98.2 \\
(34-400)\end{array}$ & $\begin{array}{c}218.9 \pm 179.1 \\
(82-530)\end{array}$ \\
\hline Controls & $(\mathrm{n}=31)$ & $\begin{array}{c}19.4 \pm 2.6 \\
(11-25)\end{array}$ & $\begin{array}{c}37.8 \pm 9.1 \\
(18-48)\end{array}$ & $\begin{array}{c}41.7 \pm 4.0 \\
(33-50)\end{array}$ & $\begin{array}{c}7.8 \pm 2.0 \\
(3-10)\end{array}$ & $\begin{array}{c}9.9 \pm 3.8 \\
(3-19)\end{array}$ & $\begin{array}{c}13.4 \pm 3.8 \\
(5-21)\end{array}$ & $\begin{array}{c}55.5 \pm 20.4 \\
(27-94)\end{array}$ & $\begin{array}{c}120.9 \pm 26.0 \\
(79-186)\end{array}$ \\
\hline
\end{tabular}

Abbreviations: Simil = WAIS Similarities; Block = WAIS Block Design; Word Tot $=$ Word List Recall Total score; Word del $=$ Word List Recall Delayed Recall; Story imm = The Babcock story immediate recall; Story del = The Babcock Story delayed recall; Serial = Serial Seven Subtraction Test time; Stroop = Stroop Test time; MNG = Meningioma; LGG = Low-grade Glioma (grade I-II); LH = Left Hemisphere; RH = Right Hemisphere. 
Table 3. Means, standard deviations and ranges of the raw scores of the neuropsychological assessment at three and 12-months follow-up.

\begin{tabular}{|c|c|c|c|c|c|c|c|c|c|}
\hline Group & & Simil & Block & Word tot & Word del & Story imm & Story del & Serial & Stroop \\
\hline MNG & $3 \mathrm{mo}$ & $\begin{array}{c}17.4 \pm 5.0 \\
(2-23)\end{array}$ & $\begin{array}{c}28.2 \pm 10.2 \\
(14-47)\end{array}$ & $\begin{array}{c}37.6 \pm 8.5 \\
(11-47)\end{array}$ & $\begin{array}{c}7.2 \pm 2.8 \\
(0-10)\end{array}$ & $\begin{array}{c}8.9 \pm 4.8 \\
(1-19)\end{array}$ & $\begin{array}{c}10.9 \pm 5.4 \\
(0-19)\end{array}$ & $\begin{array}{c}139.6 \pm 119.8 \\
(25-400)\end{array}$ & $\begin{array}{c}206.8 \pm 156.4 \\
(65-530)\end{array}$ \\
\hline \multirow{3}{*}{ LGG } & $12 \mathrm{mo}$ & $\begin{array}{c}18.0 \pm 4.4 \\
(6-23)\end{array}$ & $\begin{array}{c}30.5 \pm 8.9 \\
(12-48)\end{array}$ & $\begin{array}{c}40.3 \pm 7.9 \\
(19-49)\end{array}$ & $\begin{array}{c}7.4 \pm 2.1 \\
(1-10)\end{array}$ & $\begin{array}{c}9.5 \pm 4.6 \\
(0-20)\end{array}$ & $\begin{array}{c}11.8 \pm 5.0 \\
(0-20)\end{array}$ & $\begin{array}{c}128.8 \pm 127.9 \\
(26-400)\end{array}$ & $\begin{array}{c}194.6 \pm 158.8 \\
(65-530)\end{array}$ \\
\hline & $3 \mathrm{mo}$ & $\begin{array}{c}16.8 \pm 3.7 \\
(7-21)\end{array}$ & $\begin{array}{c}30.1 \pm 13.6 \\
(8-48)\end{array}$ & $\begin{array}{c}39.5 \pm 7.2 \\
(27-50)\end{array}$ & $\begin{array}{c}6.9 \pm 2.3 \\
(0-10)\end{array}$ & $\begin{array}{c}9.4 \pm 4.3 \\
(1-18)\end{array}$ & $\begin{array}{c}10.2 \pm 5.0 \\
(0-17)\end{array}$ & $\begin{array}{c}126.3 \pm 124.3 \\
(27-400)\end{array}$ & $\begin{array}{c}194 \pm 138.9 \\
(92-530)\end{array}$ \\
\hline & $12 \mathrm{mo}$ & $\begin{array}{c}18.1 \pm 3.7 \\
(9-22)\end{array}$ & $\begin{array}{c}34.1 \pm 10.2 \\
(18-48)\end{array}$ & $\begin{array}{c}40.8 \pm 6.7 \\
(26-50)\end{array}$ & $\begin{array}{c}7.6 \pm 1.6 \\
(4-10)\end{array}$ & $\begin{array}{c}10.4 \pm 4.6 \\
(3-17)\end{array}$ & $\begin{array}{c}10.8 \pm 4.2 \\
(1-16)\end{array}$ & $\begin{array}{c}105.9 \pm 104.6 \\
(27-400)\end{array}$ & $\begin{array}{c}210.1 \pm 156.8 \\
(84-530)\end{array}$ \\
\hline \multirow[t]{2}{*}{ Small } & $3 \mathrm{mo}$ & $\begin{array}{c}17.8 \pm 3.9 \\
(8-23)\end{array}$ & $\begin{array}{c}30.0 \pm 10.7 \\
(14-48)\end{array}$ & $\begin{array}{c}38.6 \pm 8.7 \\
(11-50)\end{array}$ & $\begin{array}{c}7.3 \pm 2.5 \\
(0-10)\end{array}$ & $\begin{array}{c}9.6 \pm 5.2 \\
(1-19)\end{array}$ & $\begin{array}{c}11.0 \pm 5.4 \\
(0-19)\end{array}$ & $\begin{array}{c}127.8 \pm 115.1 \\
(25-400)\end{array}$ & $\begin{array}{c}192.6 \pm 155.3 \\
(65-530)\end{array}$ \\
\hline & $12 \mathrm{mo}$ & $\begin{array}{c}18.4 \pm 3.5 \\
(11-23)\end{array}$ & $\begin{array}{c}31.2 \pm 9.3 \\
(12-47)\end{array}$ & $\begin{array}{c}42.3 \pm 6.7 \\
(28-50)\end{array}$ & $\begin{array}{c}7.6 \pm 2.1 \\
(1-10)\end{array}$ & $\begin{array}{c}10.1 \pm 5.1 \\
(1-20)\end{array}$ & $\begin{array}{c}12.1 \pm 5.0 \\
(0-20)\end{array}$ & $\begin{array}{c}117.2 \pm 126.5 \\
(26-400)\end{array}$ & $\begin{array}{c}187.5 \pm 154.7 \\
(65-530)\end{array}$ \\
\hline \multirow[t]{2}{*}{ Large } & $3 \mathrm{mo}$ & $\begin{array}{c}16.5 \pm 5.2 \\
(2-22)\end{array}$ & $\begin{array}{c}27.9 \pm 13.2 \\
(8-48)\end{array}$ & $\begin{array}{c}37.5 \pm 7.3 \\
(22-48)\end{array}$ & $\begin{array}{c}6.8 \pm 3.0 \\
(0-10)\end{array}$ & $\begin{array}{c}8.4 \pm 3.9 \\
(2-18)\end{array}$ & $\begin{array}{c}9.8 \pm 5.1 \\
(0-17)\end{array}$ & $\begin{array}{c}148.3 \pm 131.7 \\
(49-400)\end{array}$ & $\begin{array}{c}220.1 \pm 147.5 \\
(100-530)\end{array}$ \\
\hline & $12 \mathrm{mo}$ & $\begin{array}{c}17.9 \pm 4.8 \\
(6-23)\end{array}$ & $\begin{array}{c}32.7 \pm 10.4 \\
(16-48)\end{array}$ & $\begin{array}{c}37.9 \pm 7.7 \\
(19-49)\end{array}$ & $\begin{array}{c}7.2 \pm 1.7 \\
(4-10)\end{array}$ & $\begin{array}{c}9.7 \pm 4.3 \\
(0-16)\end{array}$ & $\begin{array}{c}10.3 \pm 4.3 \\
(0-16\end{array}$ & $\begin{array}{c}129.2 \pm 116.7 \\
(44-400)\end{array}$ & $\begin{array}{c}227.2 \pm 164.1 \\
(104-530)\end{array}$ \\
\hline \multirow[t]{2}{*}{ LH } & $3 \mathrm{mo}$ & $\begin{array}{c}17.0 \pm 5.1 \\
(2-23)\end{array}$ & $\begin{array}{c}31.2 \pm 12.7 \\
(12-48)\end{array}$ & $\begin{array}{c}37.1 \pm 9.6 \\
(11-49)\end{array}$ & $\begin{array}{c}7.1 \pm 2.8 \\
(0-10)\end{array}$ & $\begin{array}{c}7.8 \pm 4.8 \\
(1-19)\end{array}$ & $\begin{array}{c}10.0 \pm 5.5 \\
(0-19)\end{array}$ & $\begin{array}{c}133.8 \pm 114.0 \\
(27-400)\end{array}$ & $\begin{array}{c}208.9 \pm 147.6 \\
(65-530)\end{array}$ \\
\hline & $12 \mathrm{mo}$ & $\begin{array}{c}18.4 \pm 4.8 \\
(6-23)\end{array}$ & $\begin{array}{c}31.7 \pm 11.6 \\
(12-48)\end{array}$ & $\begin{array}{c}38.5 \pm 7.9 \\
(19-49)\end{array}$ & $\begin{array}{c}7.4 \pm 2.1 \\
(1-10)\end{array}$ & $\begin{array}{c}9.5 \pm 5.5 \\
(0-20)\end{array}$ & $\begin{array}{c}10.4 \pm 5.5 \\
(0-20)\end{array}$ & $\begin{array}{c}115.6 \pm 106.1 \\
(26-400)\end{array}$ & $\begin{array}{c}223.7 \pm 172.2 \\
(65-530)\end{array}$ \\
\hline \multirow[t]{2}{*}{ RH } & $3 \mathrm{mo}$ & $\begin{array}{c}17.1 \pm 4.3 \\
(7-22)\end{array}$ & $\begin{array}{c}25.9 \pm 11.4 \\
(8-48)\end{array}$ & $\begin{array}{c}39.2 \pm 6.1 \\
(27-50)\end{array}$ & $\begin{array}{c}6.9 \pm 2.8 \\
(0-10)\end{array}$ & $\begin{array}{c}10.5 \pm 4.3 \\
(4-18)\end{array}$ & $\begin{array}{c}10.5 \pm 5.0 \\
(0-17)\end{array}$ & $\begin{array}{c}128.6 \pm 131.4 \\
(25-400)\end{array}$ & $\begin{array}{c}170.2 \pm 115.1 \\
(92-530)\end{array}$ \\
\hline & $12 \mathrm{mo}$ & $\begin{array}{c}18.2 \pm 3.7 \\
(11-22)\end{array}$ & $\begin{array}{c}32.3 \pm 7.1 \\
(24-48)\end{array}$ & $\begin{array}{c}42.2 \pm 6.7 \\
(26-50)\end{array}$ & $\begin{array}{c}7.3 \pm 1.7 \\
(4-10)\end{array}$ & $\begin{array}{c}10.5 \pm 3.7 \\
(5-17)\end{array}$ & $\begin{array}{c}12.3 \pm 2.6 \\
(8-16)\end{array}$ & $\begin{array}{c}122.6 \pm 137.0 \\
(28-400)\end{array}$ & $\begin{array}{c}145.2 \pm 62.2 \\
(84-295)\end{array}$ \\
\hline \multirow[t]{2}{*}{ Anterior } & $3 \mathrm{mo}$ & $\begin{array}{c}16.3 \pm 5.2 \\
(2-23)\end{array}$ & $\begin{array}{c}26.9 \pm 11.3 \\
(8-48)\end{array}$ & $\begin{array}{c}36.8 \pm 8.2 \\
(11-49)\end{array}$ & $\begin{array}{c}6.7 \pm 3.0 \\
(0-10)\end{array}$ & $\begin{array}{c}8.6 \pm 4.3 \\
(1-19)\end{array}$ & $\begin{array}{c}9.5 \pm 5.5 \\
(0-19)\end{array}$ & $\begin{array}{c}154.0 \pm 131.0 \\
(25-400)\end{array}$ & $\begin{array}{c}244.9 \pm 178.1 \\
(65-530)\end{array}$ \\
\hline & $12 \mathrm{mo}$ & $\begin{array}{c}17.2 \pm 4.5 \\
(6-23)\end{array}$ & $\begin{array}{c}29.0 \pm 9.4 \\
(12-47)\end{array}$ & $\begin{array}{c}39.9 \pm 7.5 \\
(19-49)\end{array}$ & $\begin{array}{c}7.3 \pm 2.1 \\
(1-10)\end{array}$ & $\begin{array}{c}9.2 \pm 4.7 \\
(0-20)\end{array}$ & $\begin{array}{c}10.7 \pm 5.3 \\
(0-20)\end{array}$ & $\begin{array}{c}133.9 \pm 129.8 \\
(26-400)\end{array}$ & $\begin{array}{c}240.9 \pm 182.7 \\
(65-530)\end{array}$ \\
\hline \multirow[t]{2}{*}{ Posterior } & $3 \mathrm{mo}$ & $\begin{array}{c}18.3 \pm 1.9 \\
(16-21)\end{array}$ & $\begin{array}{c}31.7 \pm 11.4 \\
(16-48)\end{array}$ & $\begin{array}{c}41.8 \pm 7.2 \\
(26-50)\end{array}$ & $\begin{array}{c}8.0 \pm 1.7 \\
(6-10)\end{array}$ & $\begin{array}{c}10.3 \pm 4.7 \\
(3-18)\end{array}$ & $\begin{array}{c}12.3 \pm 3.7 \\
(5-17)\end{array}$ & $\begin{array}{c}100.0 \pm 96.2 \\
(46-400)\end{array}$ & $\begin{array}{c}134.2 \pm 35.9 \\
(92-195)\end{array}$ \\
\hline & $12 \mathrm{mo}$ & $\begin{array}{c}17.2 \pm 4.5 \\
(6-23)\end{array}$ & $\begin{array}{c}29.0 \pm 9.4 \\
(12-47)\end{array}$ & $\begin{array}{c}39.9 \pm 7.5 \\
(19-49)\end{array}$ & $\begin{array}{c}7.3 \pm 2.1 \\
(1-10)\end{array}$ & $\begin{array}{c}9.2 \pm 4.7 \\
(0-20)\end{array}$ & $\begin{array}{c}10.7 \pm 5.3 \\
(0-20)\end{array}$ & $\begin{array}{c}86.3 \pm 100.5 \\
(35-400)\end{array}$ & $\begin{array}{c}137.3 \pm 95.7 \\
(84-437)\end{array}$ \\
\hline
\end{tabular}

Abbreviations: Simil = WAIS Similarities; Block = WAIS Block Design; Word Tot = Word List Recall Total score; Word del = Word List Recall Delayed Recall; Story imm $=$ The Babcock story immediate recall; Story del $=$ The Babcock Story delayed recall; Serial $=$ Serial Seven Subtraction Test time; Stroop = Stroop Test time; MNG = Meningioma; LGG = Low Grade Glioma (grade I-II); LH = Left Hemisphere; RH = Right Hemisphere.

performance relative to controls with only minor exceptions in memory functions. The performance of patients with small tumor volumes was significantly hampered in attentional, visuospatial and memory functions as compared to the controls, albeit not to the same extent as observed for the large tumor group, which performed significantly lower level in all measured cognitive functions and subtests.

The patients with meningiomas performed at a significantly lower level as 
Table 4. Preoperative cognitive performance of the patient group compared to the control group.

\begin{tabular}{ccccccccc}
\hline & Simil & Block & Word tot & Word del & Story imm & Story del & Serial & Stroop \\
\hline Meningioma $(\mathrm{n}=25)$ & $\mathrm{ns}$ & $<0.001$ & $\mathrm{~ns}$ & 0.017 & $\mathrm{~ns}$ & 0.007 & $<0.001$ & 0.001 \\
LGG $(\mathrm{n}=18)$ & 0.005 & 0.021 & $\mathrm{~ns}$ & 0.034 & 0.033 & 0.002 & 0.001 & $\mathrm{~ns}$ \\
Small $(\mathrm{n}=20)$ & $\mathrm{ns}$ & $<0.001$ & $\mathrm{~ns}$ & 0.033 & $\mathrm{~ns}$ & $\mathrm{~ns}$ & 0.005 & $\mathrm{~ns}$ \\
Large (n=20) & 0.010 & 0.001 & 0.024 & 0.018 & 0.028 & $<0.001$ & $<0.001$ & 0.001 \\
LH (n=21) & $\mathrm{ns}$ & 0.006 & $\mathrm{~ns}$ & 0.050 & 0.032 & 0.004 & $<0.001$ & 0.036 \\
RH (n=14) & $\mathrm{ns}$ & $<0.001$ & $\mathrm{~ns}$ & $\mathrm{~ns}$ & $\mathrm{~ns}$ & 0.020 & 0.027 & 0.004 \\
Anterior $(\mathrm{n}=24)$ & 0.010 & $<0.001$ & $\mathrm{~ns}$ & 0.015 & 0.021 & 0.002 & $<0.001$ & 0.001 \\
Posterior $(\mathrm{n}=13)$ & $\mathrm{ns}$ & 0.042 & $\mathrm{~ns}$ & 0.049 & $\mathrm{~ns}$ & 0.007 & 0.02 & $\mathrm{~ns}$ \\
\hline
\end{tabular}

"ns" indicates no statistically significant difference between the groups on a 0.05 level. Patient groups are classified according to tumor characteristics. Abbreviations: Simil = WAIS Similarities; Block = WAIS Block Design; Word Tot $=$ Word List Recall Total score; Word del = Word List Recall Delayed Recall; Story imm = The Babcock story immediate recall; Story del = The Babcock Story delayed recall; Serial = Serial Seven Subtraction Test time; Stroop = Stroop Test time; LGG = Low-grade Glioma (grade I-II); LH = Left Hemisphere; RH = Right Hemisphere.

compared to the controls in attentional, visuospatial and delayed memory functions, while the group with low-grade gliomas (grade I-II; LGG) showed deficient performance relative to the controls in all cognitive areas with the exception of one subtest in attentional and one subtest in memory functions.

\subsection{Comparison between the Preoperative and the Post-Operative Cognitive Performance}

The results of within-patient group comparisons between the preoperative and follow-up assessment of cognitive functions are shown in Table 5 and Table 6. In the LH tumor group, no statistically significant differences emerged for any cognitive domain at the three-month follow-up when compared to preoperative assessment, but at the 12-month follow-up, significant improvements to preoperative assessment in verbal, visuospatial, attentional, and immediate memory functions were seen. Also, in the RH tumor group, significant improvement only in one subtest of immediate memory was evident at the three-month follow-up. By contrast, at the 12-month follow-up the RH tumor group showed improvement for attentional, visuospatial and delayed memory functions. The improvement seen in immediate memory at the three month follow-up no longer appeared.

At the three-month follow-up, the anterior tumor group showed improvement in visuospatial functions but deterioration in immediate memory functions, while the posterior tumor group demonstrated improvement for immediate memory functions. At the 12-month follow-up, patients with anterior tumors showed further improvement in attentional and memory functions, and the deterioration of immediate memory functions seen at the three-month follow-up did not emerge. In the posterior group, a further improvement in all but verbal functions was seen.

At the three-month follow-up, the patients with small tumor volumes exhibited improvement in visuospatial and memory functions, while those with large 
Table 5. Comparisons of cognitive performance of the tumor groups between preoperative and the three-month follow-up.

\begin{tabular}{|c|c|c|c|c|c|c|c|c|}
\hline & Simil & Block & Word tot & Word del & Story imm & Story del & Serial & Stroop \\
\hline Meningioma & ns & 0.004 & ns & 0.036 & ns & ns & ns & ns \\
\hline LGG & ns & ns & ns & ns & 0.037 & ns & ns & ns \\
\hline Small & ns & 0.033 & ns & 0.012 & ns & 0.049 & ns & ns \\
\hline Large & ns & ns & ns & ns & ns & ns & ns & ns \\
\hline $\mathrm{LH}$ & ns & ns & ns & ns & ns & ns & ns & ns \\
\hline $\mathrm{RH}$ & ns & ns & ns & ns & 0.019 & ns & ns & ns \\
\hline Anterior & ns & 0.013 & 0.027 & ns & ns & ns & ns & ns \\
\hline Posterior & ns & ns & 0.036 & ns & ns & ns & ns & ns \\
\hline
\end{tabular}

"ns" indicates no statistically significant difference between the groups on a 0.05 level. Abbreviations: Simil = WAIS Similarities; Block = WAIS Block Design; Word Tot $=$ Word List Recall Total score; Word del = Word List Recall Delayed Recall; Story imm = The Babcock story immediate recall; Story del = The Babcock Story delayed recall; Serial = Serial Seven Subtraction Test time; Stroop = Stroop Test time; LGG = Low-grade Glioma (grade I-II); LH = Left Hemisphere; $\mathrm{RH}=$ Right Hemisphere.

Table 6. Comparisons of cognitive performance of the tumor groups between preoperative and the 12-month follow-up.

\begin{tabular}{|c|c|c|c|c|c|c|c|c|}
\hline & Simil & Block & Word tot & Word del & Story imm & Story del & Serial & Stroop \\
\hline Meningioma & ns & $<0.001$ & ns & 0.019 & ns & 0.003 & 0.043 & 0.002 \\
\hline LGG & ns & ns & ns & ns & 0.001 & ns & 0.047 & ns \\
\hline Small & ns & 0.001 & ns & 0.006 & 0.041 & 0.010 & 0.007 & 0.001 \\
\hline Large & ns & 0.008 & ns & ns & 0.006 & ns & ns & ns \\
\hline $\mathrm{LH}$ & 0.030 & 0.017 & $\mathrm{~ns}$ & $\mathrm{~ns}$ & 0.006 & ns & 0.008 & $\mathrm{~ns}$ \\
\hline $\mathrm{RH}$ & ns & 0.003 & ns & ns & $\mathrm{ns}$ & 0.010 & ns & 0.002 \\
\hline Anterior & ns & $<0.001$ & ns & 0.049 & 0.038 & 0.012 & 0.027 & ns \\
\hline Posterior & ns & 0.041 & 0.016 & 0.022 & 0.032 & 0.010 & 0.028 & 0.031 \\
\hline
\end{tabular}

"ns" indicates no statistically significant difference between the groups on a 0.05 level. Abbreviations: Simil = WAIS Similarities; Block = WAIS Block Design; Word Tot $=$ Word List Recall Total score; Word del $=$ Word List Recall Delayed Recall; Story imm = The Babcock story immediate recall; Story del = The Babcock Story delayed recall; Serial = Serial Seven Subtraction Test time; Stroop = Stroop Test time; LGG = Low-grade Glioma (grade I-II); LH = Left Hemisphere; $\mathrm{RH}=$ Right Hemisphere.

tumor volumes displayed no significant change. At the 12-month follow-up, those with small tumor volumes showed further improvement in attentional, verbal and memory functions, while the patients with large tumor volumes improved their performance across visuospatial and immediate memory domains.

At the three-month follow-up, the patients with meningioma displayed improved performance in visuospatial and delayed memory functions, with the group with LGG showing enhancement in immediate memory functions. At the 12-month follow-up, the meningioma groups exhibited further improvement in delayed memory and attentional domains, with the LGG group further improving in attentional functions.

\section{Discussion}

The aims of the current study were to examine the nature of cognitive deficits in intracranial tumor patients, i.e., whether they may be local or more general in 
nature, as well as to delineate the ways in which characteristics of the tumor affect cognitive performance. In addition, the effects of surgical treatment on cognitive performance together with the factors associated with cognitive recovery were examined.

In this study, the tumor-induced cognitive impairment appeared diffuse affecting the majority of cognitive domains. However, specific cognitive deficits based on the location of the tumor were not found. Patients with anterior or large tumors suffered the most severe cognitive impairments before the surgical operation. Surgical operation of the tumor had recovering effect on tumor-induced cognitive impairment. Cognitive recovery was more noticeable in patients with meningioma as compared to those with LGG and also in patients with small tumors as compared to those with large tumors. The current results are consistent with previous evidence reporting intracranial tumor related cognitive impairment to be generalized in nature [8] [11] [12] At the same time, the present results contrast with previous evidence that has linked the location of the tumor to specific cognitive deficit [3] [4] [5] [6].

One possible explanation for the absence of clear association between the tumor location and specific type of cognitive deficit in the current study concerns the underlying neural pathology of the tumor. This seems plausible in light of previous evidence indicating that intracranial tumors cause alterations to the functional connectivity of the whole brain network when measured with magnetoencephalography [13] [28]. It has further been suggested that these functional network alterations manifest as diffuse cognitive impairment [2] [29].

In the current study, tumors with large volumes were associated with a greater negative impact on cognitive performance as compared to small tumors. This lends support to previous studies, in which large tumor volume and rapid growth rate were isolated as key factors underlying the resultant cognitive impairment [14] [15]. The mass effect of the tumor likely has a substantial impact on the nature of cognitive impairment prior to the resection of the tumor; however, the present study also suggested that patients with large tumor volumes fail recover cognitively to the same extent as do patients with smaller tumor volumes. Also, tumor volumes between meningiomas and gliomas are not interchangeable due to infiltrative nature of glioma growth, which might attenuate the effect of the tumor volume on cognitive impairment.

The surgical operation of the tumor was associated with a recovery effect on tumor-induced cognitive impairment. However, the extent of recovery was affected by the histological type of the tumor. In the current study, the patients with meningiomas showed some degree of recovery across all cognitive domains impaired at the preoperative assessment, this being in line with a review by Meskal, et al. [19]. By contrast, patients with LGG showed more modest recovery as compared to those with meningioma, albeit they too clearly benefited from the surgery. Post-surgery treatment differed significantly between these groups: while all patients with LGG except for one were treated with radiotherapy, which is a reported cause of cognitive impairment [30], only two menin- 
gioma patients received such treatment. Thus, it is possible that differences in cognitive recovery between meningioma and LGG patients are at least partially explained by the effect of radiation.

The strengths of the current study include a control group, and the design including both preoperative and postoperative neuropsychological assessments, which have been absent for the majority of existing studies on brain tumor-induced cognitive changes [19] [20]. Moreover, the current study was characterized by a respectable participation frequency, only with a few drop-outs due to death. The limitations of the current study are as follows. First, the design of the study did not allow for the potential interactions between the characteristics of the tumors, volume, malignancy and location to be explored. Second, the practice effect was not controlled for.

The current results have significant implications for the clinical practice. First, due to the diffuse nature of brain tumor-induced cognitive impairment, cognitive performance of the tumor patient needs to be assessed across all cognitive domains. Second, an important factor concerning the clinical practice is the time of the recovery. The cognitive recovery post-surgery that was observed in the current study commonly involved only partial recovery after three months, with ongoing recovery being evident at 12 months. This suggests that spontaneous recovery from the intracranial tumor operation will require a minimum of one year time-wise, which thus represents a crucial time window for rehabilitation.

In summary, intracranial tumors are linked to severe cognitive impairment in various cognitive domains. However, surgical operation of the tumor alleviates the extent of the impairment. Mitigation of the impairment and the extent and rate of cognitive recovery depend substantially both on type and the size of the tumor, as cognitive impairment appears more persistent with gliomas and large tumors. Merely a partial spontaneous recovery is observed in the few months following surgery, and recovery from the tumor and the surgery requires a minimum of one year time-wise. Further studies are warranted to examine how neuropsychological rehabilitation may alleviate tumor-induced cognitive impairment (specifically in frontal tumor patients), as well as the types of interactions that may exist between different tumor characteristics.

\section{Acknowledgements}

The authors would like to thank Riitta Herva for histological analyses and John Koivukangas for supervising the data acquisition at the Department of Neurosurgery, Oulu University Hospital.

\section{Conflicts of Interest}

The authors declare no conflicts of interest regarding the publication of this paper.

\section{References}

[1] Giovagnoli, A.R. (2012) Investigation of Cognitive Impairments in People with 
Brain Tumors. Journal of Neuro-Oncology, 108, 277-283.

https://doi.org/10.1007/s11060-012-0815-6

[2] Heimans, J.J. and Reijneveld, J.C. (2012) Factors Affecting the Cerebral Network in Brain Tumor Patients. Journal of Neuro-Oncology, 108, 231-237.

https://doi.org/10.1007/s11060-012-0814-7

[3] Hahn, C.A., Dunn, R.H., Logue, P.E., King, J.H., Edwards, C.L. and Halperin, E.C. (2003) Prospective Study of Neuropsychological Testing and Quality-of-Life Assessment of Adults with Primary Malignant Brain Tumors. International Journal of Radiation Oncology Biology Physics, 55, 992-999. https://doi.org/10.1016/S0360-3016(02)04205-0

[4] Satoer, D., Vork, J., Visch-Brink, E., Smits, M., Dirven, C. and Vincent, A. (2012) Cognitive Functioning Early after Surgery of Gliomas in Eloquent Areas. Journal of Neurosurgery, 117, 831-838. https://doi.org/10.3171/2012.7.JNS12263

[5] Emanuele, B., Santini, B., Talacchi, A., Gerosa, M. and Savazzi, S. (2012) Pre- and Post-Operative Assessment of Visuo-Spatial Functions in Right Hemisphere Tumor Patients: A Pilot Study. Journal of Neuro-Oncology, 108, 261-267.

https://doi.org/10.1007/s11060-012-0820-9

[6] Shallice, T., Mussoni, A., D’Agostino, S. and Skrap, M. (2010) Right Posterior Cortical Functions in a Tumor Patient Series. Cortex, 46, 1178-1188.

https://doi.org/10.1016/j.cortex.2010.04.005

[7] Anderson, S.W., Damasio, H. and Tranel, D. (1990) Neuropsychological Impairments Associated with Lesions Caused by Tumor or Stroke. Archives of Neurology, 47, 397-405. https://doi.org/10.1001/archneur.1990.00530040039017

[8] Noll, K.R., Ziu, M., Weinberg, J.S. and Wefel, J.S. (2016) Neurocognitive Functioning in Patients with Glioma of the Left and Right Temporal Lobes. Journal of Neuro-Oncology, 128, 323-331. https://doi.org/10.1007/s11060-016-2114-0

[9] Zucchella, C., Bartolo, M., Di Lorenzo, C., Villani, V. and Pace, A. (2013) Cognitive Impairment in Primary Brain Tumors Outpatients: A Prospective Cross-Sectional Survey. Journal of Neuro-Oncology, 112, 455-460. https://doi.org/10.1007/s11060-013-1076-8

[10] Carlesimo, G.A. (2012) Memory Disorders in Patients with Cerebral Tumors. Journal of Neuro-Oncology, 108, 253-256. https://doi.org/10.1007/s11060-012-0825-4

[11] Meskal, I., Gehring, K., Van der Linden, S.D., Rutten, G.M. and Sitskoorn, M.M. (2015) Cognitive Improvement in Meningioma Patients after Surgery: Clinical Relevance of Computerized Testing. Journal of Neuro-Oncology, 121, 617-625. https://doi.org/10.1007/s11060-014-1679-8

[12] Tucha, O., Smely, C., Preier, M. and Lange, K.W. (2000) Cognitive Deficits before Treatment among Patients with Brain Tumors. Neurosurgery, 47, 324-334. https://doi.org/10.1097/00006123-200008000-00011

[13] Bartolomei, F., Bosma, I., Klein, M., Baayen, J.C., Reijneveld, J.C., Postma, T.J., Heimans, J.J., van Dijk, B.W., de Munck, J.C., de Jongh, A., Cover, K.S. and Stam, C.J. (2006) Disturbed Functional Connectivity in Brain Tumor Patients: Evaluation by Graph Analysis of Synchronization Matrices. Clinical Neurophysiology, 117, 2039-2049. https://doi.org/10.1016/j.clinph.2006.05.018

[14] Talacchi, A., Santini, B., Savazzi, S. and Gerosa, M. (2011) Cognitive Effects of Tumor and Surgical Treatment in Glioma Patients. Journal of Neuro-Oncology, 103, 541-549. https://doi.org/10.1007/s11060-010-0417-0

[15] Brown, P.D., Jensen, A.W., Felten, S.J., Ballman, K.V., Schaefer, P.L., Jaeckle, K.A., 
Cerhan, J.H. and Buckner, J.C. (2006) Detrimental Effects of Tumor Progression on Cognitive Function of Patients with High-Grade Glioma. Journal of Clinical Oncology, 24, 5427-5433. https://doi.org/10.1200/JCO.2006.08.5605

[16] Desmurget, M., Bonnetblanc, F. and Duffau, H. (2007) Contrasting Acute and slow-Growing Lesions: A New Door to Brain Plasticity. Brain, 130, 898-914. https://doi.org/10.1093/brain/awl300

[17] Taphoorn, M.J. and Klein, M. (2004) Cognitive Deficits in Adult Patients with Brain Tumors. The Lancet Neurology, 3, 159-168. https://doi.org/10.1016/S1474-4422(04)00680-5

[18] Teixidor, P., Gatinol, P., Leroy, M., Masuet-Aumatell, C., Capelle, L. and Duffau, H. (2007) Assessment of Verbal Working Memory before and after Surgery for Low-Grade Glioma. Journal of Neuro-Oncology, 81, 305-313. https://doi.org/10.1007/s11060-006-9233-y

[19] Meskal, I., Gehring, K., Rutten, G.J.M. and Sitskoorn, M.M. (2016) Cognitive Functioning in Meningioma Patients: A Systematic Review. Journal of Neuro-Oncology, 128, 195-205. https://doi.org/10.1007/s11060-016-2115-Z

[20] Gehrke, A.K., Baisley, M.C., Sonck, A.L., Wronski, S.L. and Feuerstein, M. (2013) Neurocognitive Deficits Following Primary Brain Tumor Treatment: Systematic Review of a Decade of Comparative Studies. Journal of Neuro-Oncology, 115, 135-142. https://doi.org/10.1007/s11060-013-1215-2

[21] Folstein, M.F., Folstein, S.E. and McHugh, P.R. (1975) "Mini-Mental State": A Practical Method for Grading the Cognitive State of Patients for the Clinician. Journal of Psychiatric Research, 12, 189-198. https://doi.org/10.1016/0022-3956(75)90026-6

[22] Kleihues, P., Burger, P.C. and Scheithauer, B.W. (1993) Histological Typing of Tumors of the Central Nervous System. 2nd Edition, Springer-Verlag, Berlin. https://doi.org/10.1007/978-3-642-84988-6

[23] Wechsler, D. (1971) Wechsler Adult Intelligence Scale: A Finnish Manual. Psykologien Kustannus Oy, Helsinki.

[24] Babcock, H. and Levy, L. (1940) The Measurement of Efficiency of Mental Functioning (Revised Examination): Test and Manual of Directions. C.H. Stoelting, Chicago, IL.

[25] Christensen, A.L. (1979) Luria's Neuropsychological Investigation. Munksgaard, Copenhagen.

[26] Golden, C. (1978) Stroop Color and Word Test. Stoelting Company, Illinois.

[27] Lezak, M.D. (1995) Neuropsychological Assessment. 3rd Edition, Oxford University Press, New York, NY.

[28] Bosma, I., Douw, L., Bartolomei, F., Heimans, J.J., van Dijk, B.W., Postma, T.J., Stam, C.J., Reijneveld, J.C. and Klein, M. (2008) Synchronized Brain Activity and Neurocognitive Function in Patients with Low-Grade Glioma: A Magnetoencephalography Study. Neuro-Oncology, 10, 734-744. https://doi.org/10.1215/15228517-2008-034

[29] Derks, J., Reijneveld, J.C. and Douw, L. (2014) Neural Network Alterations Underlie Cognitive Deficits in Brain Tumor Patients. Current Opinion in Oncology, 26, 627-633. https://doi.org/10.1097/CCO.0000000000000126

[30] Greene-Schloesser, D. and Robbins, M.E. (2012) Radiation-Induced Cognitive Impairment-From Bench to Bedside. Neuro-Oncology, 14, 37-44.

https://doi.org/10.1093/neuonc/nos196 